\title{
Complete remission of coronary vasculitis in Churg-Strauss Syndrome by prednisone and cyclophosphamide
}

\author{
Niels P. Riksen • Helmut Gehlmann • \\ Annemarie E. Brouwer • Marcel van Deuren
}

Received: 11 December 2009 /Revised: 2 March 2010 / Accepted: 3 March 2010 / Published online: 28 March 2010

(C) The Author(s) 2010. This article is published with open access at Springerlink.com

\begin{abstract}
The heart is involved in up to $50 \%$ of all patients with Churg-Strauss syndrome, but vasculitis of the coronary arteries has only been rarely documented. We present a young patient with severe coronary aneurysms and stenotic lesions due to a Churg-Strauss vasculitis. Prompt therapy with prednisone and cyclophosphamide resulted in the complete resolution of all lesions.
\end{abstract}

Keywords Churg-Strauss syndrome - Coronary vasculitis . Cyclophosphamide $\cdot$ Prednisone

\section{Introduction}

The heart is involved in up to $50 \%$ of all patients with ChurgStrauss disease $[1,2]$. In contrast, vasculitis of the coronary arteries has only been rarely documented [3-5]. Here, we present images of repeated coronary angiography in a young

N. P. Riksen $(\bowtie)$

Department of Internal Medicine, Division of Vascular Medicine, Radboud University Nijmegen Medical Center,

Geert Grooteplein 21, PO Box 9101, 6500 HB Nijmegen,

The Netherlands

e-mail: N.Riksen@aig.umcn.nl

H. Gehlmann

Department of Cardiology,

Radboud University Nijmegen Medical Centre,

Nijmegen, The Netherlands

\section{A. E. Brouwer · M. van Deuren}

Department of Internal Medicine and Nijmegen Institute

for Infection, Inflammation and Immunity (N4i),

Radboud University Nijmegen Medical Centre,

Nijmegen, The Netherlands patient with Churg-Strauss syndrome demonstrating complete resolution of severe coronary vasculitis after 1 year of therapy with prednisone and cyclophosphamide.

\section{Case report}

An 18-year-old man with a history of asthma was admitted to a local hospital because of fever, profound headache, weight loss, red eyes, blurred vision, and generalized muscle aches. All symptoms resolved spontaneously within 1 month before a classifying diagnosis could be made. Five months later, the headache and blurred vision recurred, and the patient was referred to our outpatient clinic. Laboratory analysis at that moment revealed only mild eosinophilia $\left(11 \% ; 600 \times 10^{6} / \mathrm{L}\right)$. Renal function was normal, and antineutrophilic cytoplasmatic antibodies and anti-nuclear antibodies were not present. Cranial magnetic resonance imaging showed a unilateral thickening of the choroidal cerebral plexus, and analysis of the cerebrospinal fluid revealed eosinophilic meningitis. Another 3 months later, the patient developed a persistent fever and a pulmonary infiltrate that did not respond to antibiotics. Bronchoalveolar lavage revealed profound eosinophilia (30\%). During admission, eosinophilic skin infiltrates and generalized edema developed. Now, we considered the diagnosis of Churg-Strauss syndrome, and therapy with prednisone at a dose of $1 \mathrm{mg} / \mathrm{kg}$ was initiated. Subsequently, the patient developed a painful injected pharynx and chest pain, with diffuse ST segment elevation on electrocardiography. Echocardiopgraphy revealed a mild pericardial effusion, without wall motion abnormalities. Intravenous gammaglobulin (400 mg/kg per day for 5 days) was now administered. As the chest pain recurred and there was a rise in the plasma troponin-I concentration, a coronary 


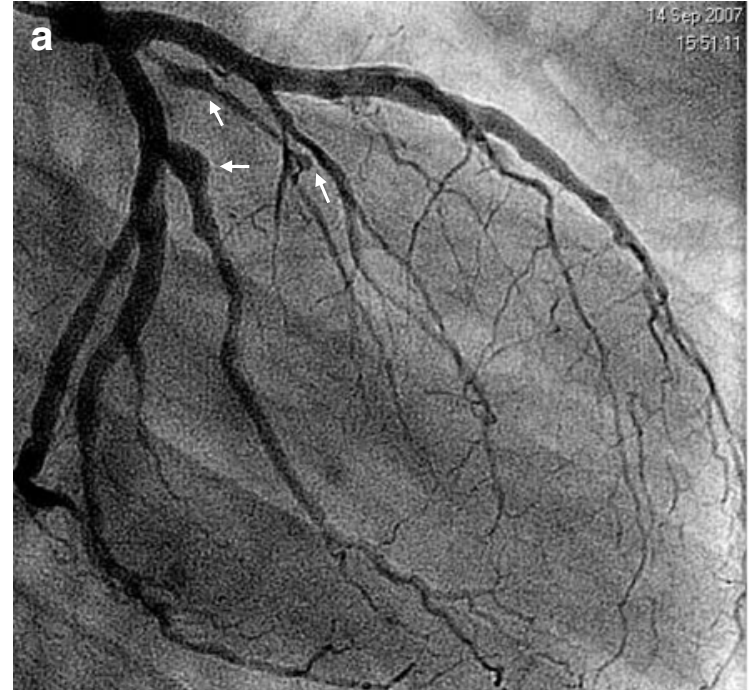

Fig. 1 Left coronary angiogram in right inferior oblique view before (a) and after (b) 1 year of immunosuppressive therapy with prednisone and cyclophosphamide. Before the treatment, there were two prominent aneurysms in the intermediate branch and one large

angiography was performed. This showed multiple aneurysms and stenotic lesions in all coronary arteries (Fig. 1a). MRI scanning excluded involvement of the aorta and its major branches. In view of the potentially fatal coronary lesions, a more powerful immune-suppressive regimen consisting of methylprednisolone and cyclophosphamide (750 $\mathrm{mg}$ and 1,250 $\mathrm{mg}$ i.v. per day during 3 days, followed by oral doses of $1 \mathrm{mg} / \mathrm{kg} /$ day prednisone, and $2 \mathrm{mg} / \mathrm{kg} /$ day of cyclophosphamide) was chosen. The prednisone was tapered gradually and stopped after 1 year. The cyclophosphamide was tapered and stopped after 16 months. A control coronary angiography 1 year after initiation of therapy showed a complete regression of all lesions (Fig. 1b).

Our patient fulfilled the classification criteria of the Churg-Strauss syndrome as established by the American College of Rheumatology (asthma, eosinophilia, pulmonary infiltrates, and a biopsy containing accumulation of eosinophils), although he also had signs and symptoms compatible with other vasculitis syndromes, such as Kawasaki disease (bilateral conjunctival injection, injected pharynx).

Endocardial thickening due to local infiltration of eosinophils, which can trigger intracardial thrombus formation, is the most common presentation of heart involvement in patients with the Churg-Strauss syndrome [1, 2]. Pericardial involvement, presenting either as an acute pericarditis with pericardial effusion, as in our patient, or as chronic constrictive pericarditis, has been reported in 8 $32 \%$ of patients [1]. In contrast, clinically significant

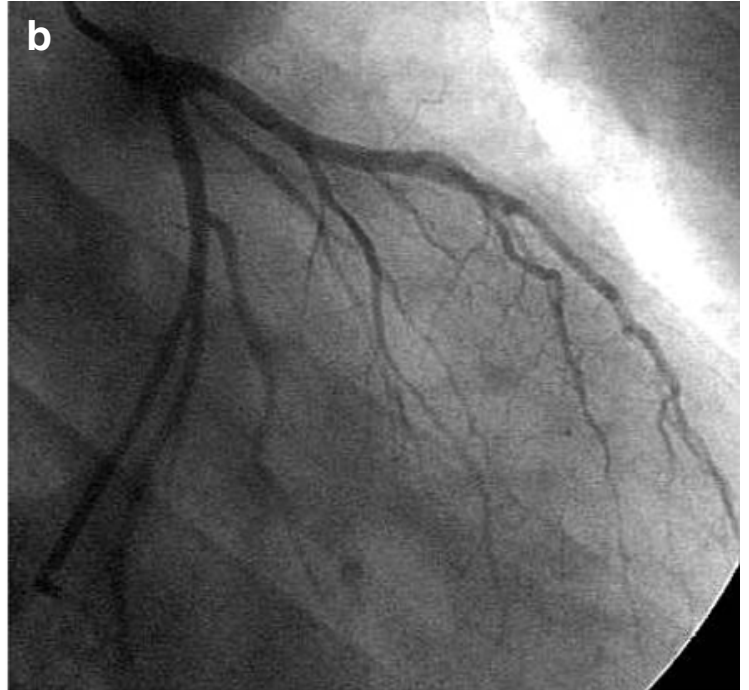

aneurysm proximally in the obtuse marginal branch (a, indicated by white arrows) in addition to diffuse wall irregularities in all branches. After the treatment, all these lesions have disappeared completely (b)

vasculitis of the coronary arteries has been reported only anecdotically [3-5]. In general, symptomatic cardiac involvement is associated with poor prognosis in patients with medium or small vessel vasculitis and justifies a strong and immediate immunosuppressive treatment. Indeed, the present case report shows that therapy with prednisone and cyclophosphamide may lead to a complete and uneventful recovery of potentially fatal coronary artery lesions.

\section{Disclosures None}

Open Access This article is distributed under the terms of the Creative Commons Attribution Noncommercial License which permits any noncommercial use, distribution, and reproduction in any medium, provided the original author(s) and source are credited.

\section{References}

1. Kane GC, Keogh KA (2009) Involvement of the heart by small and medium vessel vasculitis. Curr Opin Rheumatol 21:29-34

2. Churg J, Strauss L (1951) Allergic granulomatosis, allergic angiitis, and periarteritis nodosa. Am J Pathol 27:277-301

3. Hellemans S, Dens J, Knockaert D (1997) Coronary involvement in the Churg-Strauss syndrome. Heart 77:576-578

4. Kozak M, Gill EA, Green LS (1995) The Churg-Strauss syndrome. A case report with angiographically documented coronary involvement and a review of the literature. Chest 107:578-580

5. Drogue M, Vergnon JM, Wintzer B, Antoine JC, Malquerti V (1993) Prinzmetal's angina pectoris revealing aneurysm of the right coronary artery during evolution of Churg-Strauss syndrome. Chest 103:978 